\title{
HIGHLIGHTS
}

IMMUNOTHERAPY

\section{A promising vaccine for glioblastoma multiforme}

A vaccine that targets $E G F R$ variant III

(EGFRvIII) can improve survival

in patients with newly diagnosed

glioblastoma multiforme, Sampson and

colleagues report. The vaccine induces

mutation-specific immunity in patients

with EGFRvIII-positive glioblastoma

multiforme and may be linked to the

eradication of cells expressing EGFR $I I I$ at

disease recurrence.

Despite the availability of aggressive treatment modalities, patients with glioblastoma multiforme have a median overall survival of just 14.6 months. Targeting tumor-specific mutations with an immunological

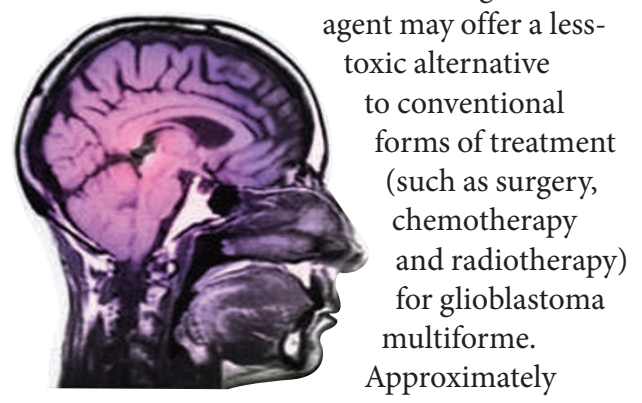

one-third of these tumors are reported to express EGFRvIII, a mutant cell-surface protein that is associated with the growth and migration of tumors, making it an ideal target for immunotherapy.

Preclinical data have indicated that an EGFRvIII-targeted vaccine has activity in glioblastoma multiforme. These findings formed the basis for the latest investigation by Sampson and coworkers. The researchers assessed 35 patients with EGFRvIII-positive glioblastoma multiforme treated with radiation therapy and temozolomide, 18 of whom also received the vaccine.

Compared with the control arm, patients treated with the vaccine had a better progression-free survival (6.3 months versus 14.2 months) and a longer overall survival (median 15 months versus 26 months). The vaccine was generally well-tolerated with no toxic effects exceeding grade 2 severity.

Of note, survival was improved in those patients who experienced an immune response compared with those who did not. Most recurrent tumors in the patients treated with the vaccine did not have any EGFRvIII-expressing cells, suggesting that the

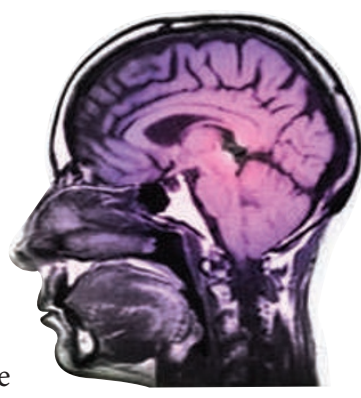
tumors may have mutated to become resistant to the vaccine.

Despite these promising data, the investigators recommend that a larger randomized study is needed to confirm the efficacy of this strategy. Tumor-specific vaccines are promising agents as they limit autoimmune complications. However, the authors note that targeting only one tumor-specific antigen may have limited benefits for cancers with multiple antigens.

\section{Lisa Richards}

Original article Sampson, J. H. et al. Immunologic escape after prolonged progression-free survival with epidermal growth factor receptor variant III peptide vaccination in patients with newly diagnosed glioblastoma. J. Clin. Oncol. 28, 4722-4729 (2010) 